\title{
A Case Report of Tetralogy of Fallot Associated with Tight Lesion of the Left Anterior Descending Coronary Artery
}

\author{
J Kabir, R Rima, R Hassan, AMM Badruddoza, S Khatoon
}

Keywords: Tetralogy of Fallot, Left anterior descending artery (LAD), CABG.

\begin{abstract}
:
A small subset of patients with TOF present later in life with unsuspected or untreated disease. Tetralogy of Fallot in adults represents a special group with peculiar problems related to the effects of prolonged cyanosis over the heart and other organs. Older age has been considered as incremented risk factor for surgical mortality and long-term survival in patients with TOF. Based on this assumption, indication for surgery after long-standing cyanosis has caused controversy. There are few reports that studied this issue have proved some benefit in repairing adults with TOF .The greatest benefit of complete repair at this age is the functional improvement. We report a male patient with TOF with tight lesion of the left anterior descending coronary artery diagnosed at age 40 years and successfully underwent total correction along with CABG. This patient was the oldest documented in Bangladesh undergoing successful corrective surgery for TOF with CABG.
\end{abstract}

(Cardiovasc.j. 2011; 4(1): 88-91)

\section{Introduction:}

Tetralogy of Fallot (TOF), first described in 1888 is a disease consisting of malalignment of the outlet septum-ventricular septal defect (VSD), aortic override, pulmonary outflow tract stenosis and right ventricular hypertrophy. It is the most common form of cyanotic congenital heart disease ( $10 \%$ of all cases). It is common in pediatric congenital heart disease but rare in un-operated adult especially those older than 40 years of age. Epidemiological studies and autopsy records demonstrated that approximately $2 \%$ of all patients with tetralogy of Fallot (TOF) reach the fourth decade of life. ${ }^{1}$

The survival rate of patients who receive repair surgery is about $86 \%$ at 32 years follow-up and $85 \%$ at 36 years follow-up survival rates of unoperated TOF patients older than 10 years is about $30 \%$, older than 20 years $11 \%$, older than 30 years $6 \%$ and older than 40 years only about $3 \%{ }^{2}, 3$

We report a male patient with TOF diagnosed at age 40 years associated with tight lesion of the left anterior descending coronary artery and successfully operated at a single stage. This patient was the oldest documented publicly in Bangladesh undergoing successful corrective surgery for TOF.

\section{Case Report:}

A 40-year-old male patient presented with exercise intolerance (NYHA class II), fatigue for last 7-8 years. He had retrosternal chest pain one and half year ago for which he got admitted in hospital and was treated with low molecular weight heparin. Since then he is on aspirin and beta blocker. Physical examination revealed blood pressure 110/ $70 \mathrm{~mm} \mathrm{Hg}$, regular pulse $96 \mathrm{bpm}$ and respiratory rate 22 breaths $/ \mathrm{min}$. Mild cyanosis and clubbing of the fingers were noted. The lungs were clear and a systolic ejection murmur grade $3 / 6$ at left sternal border (LSB) was noted. No lower limb edema was noted. There was no hepatosplenomegaly.

Laboratory data revealed WBC 6880/dL, Hemoglobin 17.5 g/dl, Hct 57.3\%, PLT 125,000/dL. Blood urea, serum Creatinine \& serum electrolytes are normal. Lipid profile showed Serum total cholesterol 291.6 $\mathrm{mg} / \mathrm{dl}$, LDL $149.4 \mathrm{mg} / \mathrm{dl}$, HDL $22.4 \mathrm{mg} / \mathrm{dl}$, Triglyceride $1263 \mathrm{mg} / \mathrm{dl}$. The chest x-ray revealed cardiomegaly and relative oligaemic bilateral lung fields. The 12-lead electrocardiogram demonstrated a right ventricular hypertrophy with strain pattern. Echocardiography revealed anatomy consistent with tetralogy of Fallot with severe infundibular and valvular pulmonary stenosis with a pressure gradient

Address of correspondence: Dr. Rezoana Rima, Specialist Paediatric Cardiology, United Hospital Limited, Dhaka,E mail: rezoanabd@yahoo.com 
of $84 \mathrm{mmHg}$ and hypoplastic main pulmonary artery (MPA), stenosis also involves confluence and LPA origin. A large interruption of the inter-ventricular septum (IVS) at the subaortic portion, sized $2.1 \mathrm{~cm}$ with bi-directional shunt and 40-50\% aortic overriding was observed. Cardiac catheterization demonstrated a subaortic VSD, infundibular and valvular pulmonary stenosis with formation of third chamber and right ventricular enlargement, and hemodynamic data revealed that the right ventricular $(\mathrm{RV})$ pressure $(130 / 15 \mathrm{mmHg})$ was almost equal to the left ventricular (LV) pressure (133/13 mmHg). Coronary artery angiogram showed tight lesion in proximal part of LAD.

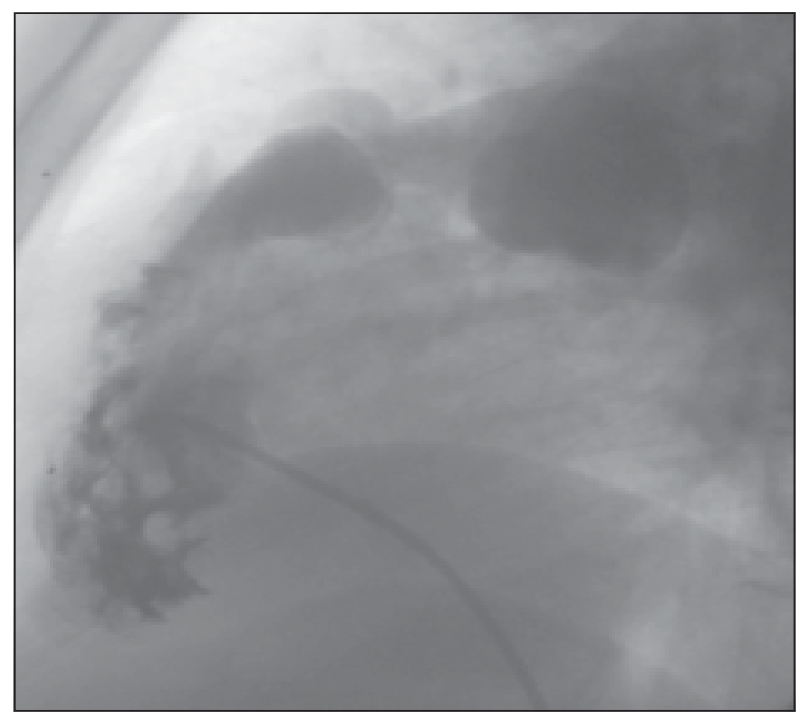

Fig.-1: $R V$ angiogram at lateral view showed infundibular stenosis, dome shaped pulmonary valve.

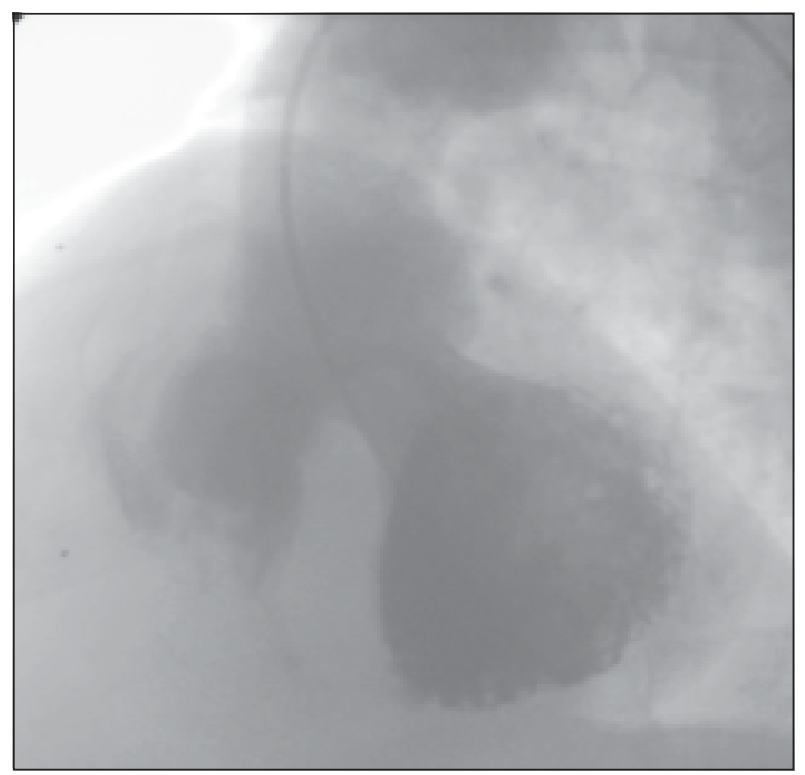

Fig.-2: LV angiogram showed large subaortic VSD.

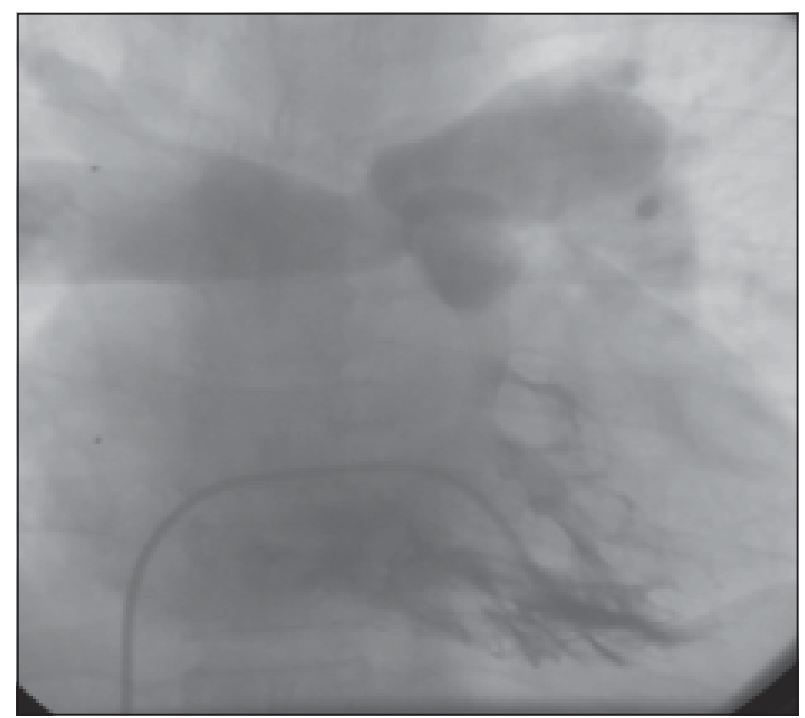

Fig.-3: $R$ V angiogram at AP Cranial view showed thickened pulmonary valve, confluence stenosis (Type II PPS).

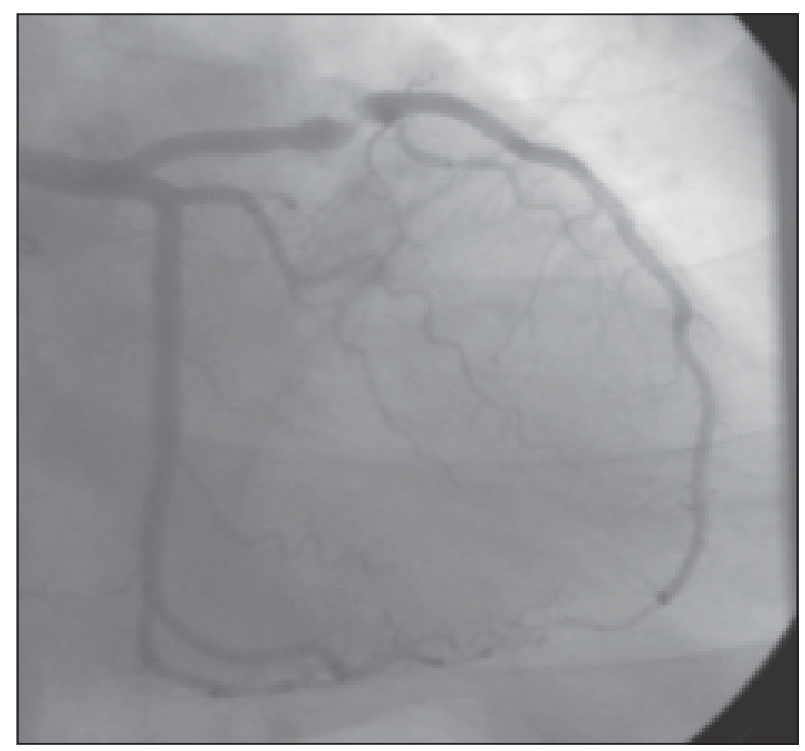

Fig.-4: $C A G$ showed tight (90\%) stenosis at proximal part of $L A D$.

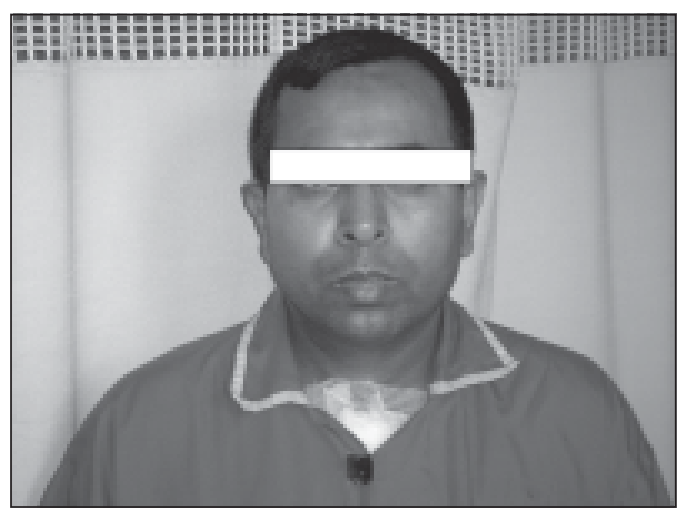


The patient underwent total repair of TOF and CABG (LIMA to LAD) at one stage. At first CABG was done and followed by total repair of TOF which includes dacron patch closure of VSD, resection of RVOT band, valvular stenosis was dilated with artery forceps and Hager's dilator upto no. 20. Separate pericardial patch augmentation of RVOT and main pulmonary artery were done. But patient could not be weaned from bypass and was found high RV pressure and significant gradient across RVOT and PA. So again bypass established, transannular patch augmentation upto LPA was done and gradual weaning from cardiopulmonary bypass was achieved. At the end of the procedure RV pressure was two third of systemic pressure and there was no significant stenosis across RVOT and MPA. Total cardiopulmonary bypass time was 248 minutes and total cross clamp time was 139 minutes. Patient came from OT with minimum ionotropic support and was extubated on $1^{\text {st }}$ POD. His postoperative period was uneventful except he required prolonged chest drainage because of $R V$ failure due to RV systolic and diastolic dysfunction. His postoperative echo showed tiny residual VSD, mild LPA origin stenosis, impaired relaxation of $R V$, fair LV systolic function. His exercise tolerance (NYHA class I) was improved a lot at follow up; no other specific events were noted at the outpatient department visits after about 18 months followup.

\section{Discussion:}

TOF is rarely detected in a 40-year-old patient. It may be difficult to make a correct diagnosis by history taking, physical examination, ECG and chest X Ray. Echocardiography is a good tool for diagnosis and evaluation but the examiner should maintain a high index of suspicion. Our patient also incidentally diagnosed as TOF while evaluating for retrosternal pain.

Complete surgical correction is now the most important and standard treatment of TOF. Mortality is about 3\% in children and 2.5\% to $8.5 \%$ in adults. ${ }^{4}$ Several factors, such as old age, high hemoglobin level, pulmonary artery hypoplasia and a diminutive left ventricle, have been identified as risk factors for operative mortality in many previously published series. ${ }^{5}$

Cyanotic congenital heart diseases constitute about 10 percent of total congenital heart disease cases in adults in the developing world. ${ }^{6}$ Prolonged cyanosis and old age adversely affect the outcome of surgery, thus posing a challenge to the cardiac surgeons. The overall survival of surgically treated adult patients with TOF is acceptable and the great benefit of complete repair at this age is the functional improvement. ${ }^{7}$ Mayo Clinic experience (June 1960 to May 1982) with 30 patients of 40 to 60 years old (mean 47) who had total surgical correction of tetralogy of Fallot was reviewed. At follow-up of 5 to 266 months; of the 22 patients who survived, 16 are in NYHA class I, 5 are in NYHA class II and 1 is in NYHA class III. ${ }^{8}$ Our patient also improves exercise tolerance (NYHA class I) after surgical repair. There is still no alternative to an early operation. Late complications such as arrhythmia and ventricular dysfunction are closely related to chronic hypoxia and might need a more careful follow-up after surgical correction. ${ }^{7}$ The common complications after repair are endocarditis, LV dysfunction, RV dysfunction, exercise intolerance, heart block, sudden cardiac death (SCD) and arrhythmia. Our patient also developed RV dysfunction after surgery.

Koymoto Y described A 31-year-old male with tetralogy of Fallot (TF) and total occlusion of the right coronary orifice complicated with infective endocarditis successfully underwent total repair of TOF and coronary artery bypass graft (CABG). ${ }^{9}$

In summary, a TOF patient diagnosed at age 40 is rare. Total correction of tetralogy of Fallot along with CABG can be performed safely in adults with low mortality and good functional improvement. Surgical repair is the definite therapy though some potential complications should be watched for and carefully followed up.

\section{References:}

1. Kirklin JW, Barratt-Boyes BG. Cardiac surgery: morphology, diagnostic criteria, natural history, techniques, results, and indications. 2nd ed. New York, NY: Churchill Livingstone; 1993: 946-1073.

2. Murphy JG, Gersh BJ, Mair DD, et al: Long-term outcome in patients undergoing surgical repair of tetralogy of Fallot. N Engl J Med 1993; 329: 593-599.

3. Brickner ME, Hillis LD, Lange RA: Congenital heart disease in adults. Second of two parts. $N$ Engl J Med 2000; 342: 334-342. 
4. Presbitero P, Prever SB, Contrafatto I, Morea M: As originally published in 1988: Results of total correction of tetralogy of Fallot performed in adults. Ann Thorac Surg 1996; 61:1870-1873.

5. Mohanty SR, Airan B, Bhan A, Sharma R, Kumar AS, Kothari SS, Saxena A, Venugopal P. Adult cyanotic congenital heart disease: surgical experience. Indian Heart J 1999; Mar-Apr;51(2):186-92.

6. S. Y. Chan, J. J. Hwang, K. L. Hsu, et al: Patient with Tetralogy of Fallot Operated at Older Age - A Case Report and Literature Review. Tzu Chi Med J. 2006; 18:121-124.
7. Fernando A Atik, Edmar Atik, Claudio R da Cunha: Longterm results of correction of tetralogy of Fallot in adulthood. European Journal of Cardio-thoracic Surgery 2004; 25: 250-255.

8. DC Hu, JB Seward, FJ Puga, V Fuster, and AJ Tajik :Total correction of tetralogy of Fallot at age 40 years and older: long-term follow-up. J Am Coll Cardiol 1985; 5:40-44.

9. Koumoto Y, Ikawa S, Shimazaki Y, Kadoba K, Matsuda H, Kawashima Y: [ case report of tetralogy of Fallot associated with total occlusion of the right coronary artery and complicated with infective endocarditis]. Nippon Kyobu Geka Gakkai Zasshi 1992; 40(11): 2082-6. 